\title{
Commentary Psychiatric Stress Testing: Novel Strategy for Translational Psychopharmacology
}

\author{
Stephen M Stahl*1,2 \\ 'Department of Psychiatry, University of California, Carlsbad, CA, USA; ${ }^{2}$ Department of Psychiatry, University of Cambridge, Cambridge, UK
}

Neuropsychopharmacology (2010) 35, |4I3-|4|4; doi:I0.1038/npp.20 I0.29

A major focus of clinical neuroscience research today is to elucidate how reactions of brain circuits to stressful loads are regulated by genes and by psychiatric disorders. The study of Salvadore et al (2010) provides a glimpse into how such 'psychiatric stress tests' may also translate into predictors of who will respond to a specific drug.

\section{WHAT IS PSYCHIATRIC STRESS TESTING?}

Numerous mental tasks can put stress upon specific brain circuits. Information processing by these circuits following such a provocative load can then be quantified using various imaging (Fineberg et al, 2010; Stahl, 2008; Bush et al, 2005) or electrophysiological techniques (Salvadore et al, 2009, 2010). The $N$-back cognitive load, for example, is a 'stress test' generally used to tax the functioning of the dorsolateral prefrontal cortex (DLPFC); the Stroop task tests the function of the dorsal anterior cingulate cortex; facial expressions test the amygdala and perigenual anterior cingulate cortex (pgACC); gambling tasks test the orbital frontal cortex; and so on (Salvadore et al, 2009, 2010; Fineberg et al, 2010; Stahl, 2008; Bush et al, 2005). The reaction of a brain circuit to the specific load placed upon it can be used as a measure of the efficiency of information processing in that brain circuit.

\section{STRESS TESTING FOR PSYCHIATRIC SYMPTOMS IN PSYCHIATRIC DISORDERS}

Applying a functional load on various brain circuits in patients with a wide range of psychiatric disorders suggests that inefficient information processing (too high or too low) may map specific symptoms to specific malfunctioning circuits. For example, cognitive symptoms such as problemsolving difficulties and trouble concentrating may map to

\footnotetext{
*Correspondence: Dr SM Stahl, Department of Psychiatry, University of California, Neuroscience Education Institute, Suite 103, 1930 Palomar Point Way, Carlsbad, CA 92008, USA, Tel: + I 760444 9903, Fax: + | 760931 85 I7, E-mail: smstah|@neiglobal.com

Received 17 February 2010; accepted 17 February 2010
}

the DLPFC, whereas emotional symptoms such as mood and anxiety map to the amygdala and pgACC (Salvadore et al, 2009, 2010; Fineberg et al, 2010; Stahl, 2008; Bush et al, 2005). As the brain has a limited number of circuit 'highways' on which it can carry its symptoms, it is not surprising that patients with the same symptoms but who have different psychiatric disorders may nevertheless express the same inefficiencies of information processing in the same circuits (eg, problems concentrating in attention-deficit hyperactivity disorder, in major depression, and anxiety disorders may all map to DLPFC; Fineberg et al, 2010; Stahl, 2008; Bush et al, 2005). Such findings are leading the charge away from the classical categorical approach for diagnosing psychiatric disorders in DSM IV to a dimensional approach for diagnosing psychiatric disorders with symptoms that may cut across numerous psychiatric disorders, although that approach will not be ready in time for DSM V. Nevertheless, the field is furiously trying to define appropriate stress-testing paradigms in order to uncover the circuit(s) of postulated inefficient information processing across the landscape of psychiatric symptoms and to objectify their biological basis and pathophysiology. Although this is a formidable task, it is already possible to see that stress testing using a quantitative measurement of brain circuit functioning has the potential to redefine psychiatric disorders.

\section{CAN PSYCHIATRIC STRESS TESTING PREDICT TREATMENT RESPONSE?}

The findings of Salvadore et al (2010) now provide insight into how these same psychiatric stress testing paradigms might also be used to predict treatment responses in individual patients. These investigators have focused on the rapid antidepressant response to a single intravenous infusion of the $N$-methyl-D-aspartate antagonist ketamine in patients with major depression. They have also looked at the pre-treatment functional responses of the pgACC to various types of psychiatric stress testing in depressed patients who receive this treatment. Previously, this group has suggested that the ability of the pgACC to react 
functionally to an affective load (ie, to fearful faces) identifies those patients who will respond to ketamine (Salvadore et al, 2009). The current study by the same authors (Salvadore et al, 2010) now suggests that the lack of response of the pgACC to a cognitive load (ie, to the $\mathrm{N}$-back test) also identifies those patients who will respond to ketamine. Normally the pgACC should not react to the $\mathrm{N}$-back test, but in some depressed patients this cognitive load nevertheless seems to activate the emotional circuit including the pgACC; these are the patients who apparently do not respond to ketamine in the Salvadore et al (2010) study. Their results using two stress tests to identify a predictive biomarker of treatment response to ketamine in depression can be summarized as different loads, same brain area, and opposite functional reactions, both correlating with treatment response to ketamine (Salvadore et al, 2009, 2010).

Obviously, these observations require independent replication, and there are many confounds that must be sorted out. For example, the patients in the Salvadore et al (in press) study had various degrees of resistance to prior treatment with other antidepressants and variability in the presence and type of comorbid anxiety disorder. In addition, the open-label design cannot separate drug effects from placebo effects. Furthermore, preliminary indications from similar investigations by others show the involvement of the pgACC in the clinical response to antidepressants of many different mechanisms, to cognitive behavioral therapy, to sleep deprivation, and probably even to placebo (Salvadore et al, 2010). Therefore, the effects described by Salvadore et al (2010) may not be linked specifically to a glutamate mechanism.

The promise of psychiatric stress tests as potential biomarkers of treatment response comes just as psychopharmacology is celebrating its 50th anniversary, amidst a dearth of novel therapeutics and with current clinical trials suddenly beset with problems showing drug-placebo differences, even for proven therapeutics. To get past these difficulties and to reignite the discovery of more effective therapeutics in psychopharmacology, objective biomarkers are needed to determine who will respond to a drug of a given mechanism, and who will not. With skyrocketing placebo response rates now being reported in a current epidemic of failed clinical trials in psychopharmacology, the field also needs a reliable biomarker to determine who will respond to placebo. The observations of Salvadore et al (2010) suggest a new research strategy to facilitate the rapid translation of novel psychopharmacological mechanisms into effective clinical therapeutics, namely by developing predictors of individuals who will respond to novel mechanisms. This approach could even guide the selection of patients who should receive treatment in large clinical trials and even help to unravel the mystery of placebo responders in psychopharmacology. Although the study of Salvadore et al (2010) may raise more questions than answers, it provides a useful beacon for translational psychopharmacology.

\section{DISCLOSURE}

Over the past 12 months (January 2009-January 2010), Dr Stahl has served as a Consultant to Allergan, Astra Zeneca, BioMarin, BioVail, Boehringer Ingelheim, Bristol MyersSquibb, Cenerex, Covance, Cypress Bioscience, Dainippon Sumitomo, Eisai, Eli Lilly, Forest, GlaxoSmith Kline, Labopharm, Lundbeck, Marinus, Meda Corp, Meiji, Merck, Novartis, Pfizer, Pfizer Canada, Pierre Fabre, PamLab, Prexa Pharmaceuticals, Propagate Pharma, Royalty Pharma, Sanofi, Schering Plough Corporation, Shire, SK Corporation, Soffinova, Solvay, Vanda, and Wyeth. He has served on the speaker's bureaus for Pfizer, Wyeth Pharmaceuticals, and Schering Plough Corporation, and has received research and/or grant support from Astra Zeneca, Boehringer Ingelheim, Bristol Myers-Squibb, Cephalon, Dainippon Sumitomo, Eli Lilly, Forest, Lundbeck, Novartis, PamLabs, Pfizer, Pfizer Canada, Pharmasquire, Sanofi Aventis, Schering Plough, Shire, and Wyeth.

\section{REFERENCES}

Bush G, Valera EM, Seidman LJ (2005). Functional neuroimaging of attention-deficit/hyperactivity disorder: a review and suggested future directions. Biol Psychiatry 57: 1273-1284.

Fineberg NA, Potenza MN, Camberlain SR, Berlin HA, Menzies L, Bechara A et al (2010). Probing compulsive and impulsive behaviors, from animal models to endophenotypes: a narrative review. Neuropsychopharmacology 35: 591-604.

Salvadore G, Cornwell BR, Colon-Rosario V, Coppola R, Grillon C, Zarate Jr CA et al (2009). Increased anterior cingulate cortical activity in response to fearful faces: a neurophysiological biomarker that predicts rapid antidepressant response to ketamine. Biol Psychiatry 65: 289-295.

Salvadore G, Cornwell BR, Sambatoro F, Latov D, Colon-Rosario V, Carver F et al (2010). Anterior cingulate desynchronization and functional connectivity with the amygdala during a working memory task predict rapid antidepressant response to ketamine. Neuropsychopharmacology. (in press)

Stahl SM (2008). Stahls Essential Psychopharmacology 3rd edn. Cambridge University Press: New York. 